\title{
Different Associations between High Density Lipoprotein Cholesterol and Cardiovascular Diseases in Diabetic and Non-diabetic People: A Prospective Community-based Study
}

Zhijun Wu ( $\nabla$ totito19822005@126.com )

Shanghai Jiao Tong University Medical School Affiliated Ruijin Hospital

Zhe Huang

Kailuan General Hospital

Alice H. Lichtenstein

Tufts University

Cheng Jin

Kailuan General Hospital

Shuohua Chen

Kailuan General Hospital

Frank B Hu

Harvard University

Shouling Wu

Kailuan General Hospital

\section{Xiang Gao}

The Pennsylvania State University https://orcid.org/0000-0003-2617-6509

Original investigation

Keywords: high density lipoprotein cholesterol, diabetes mellitus, myocardial infarction, prospective study

Posted Date: July 9th, 2020

DOI: https://doi.org/10.21203/rs.3.rs-37482/v1

License: (c) (i) This work is licensed under a Creative Commons Attribution 4.0 International License. Read Full License 


\section{Abstract}

Background: Experimental studies found that the functionality of high density lipoprotein cholesterol (HDL-C) may be lost in the presence of diabetes mellitus (DM). We prospectively tested whether DM modified the association between HDL-C concentrations and cardiovascular disease (CVD).

Methods: Included were 91,354 Chinese adults (8,244 participants with DM and 83,110 participants without DM) without use of lipid-lowering drugs and free of CVD or cancer at baseline (2006). The primary endpoint of interest was a composite of CVD (myocardial infarction, ischemic stroke and hemorrhagic stroke). Cumulative average HDL-C concentrations were calculated from all available HDL-C measures at baseline (2006) and during the follow-up period (2008, 2010, 2012 and 2014).

Results: During a mean of 10.4 year of follow-up, there were 5,076 CVD events identified. Presence of DM significantly modified the association between HDL-C concentrations and CVD risk $\left(P_{\text {interaction }}=0.003\right)$. The association between HDL-C concentrations and CVD followed a U-shaped curve in individuals without DM ( $\left.\mathrm{P}_{\text {nonlinearity }}<0.001\right)$. The adjusted hazard ratio $(H R)$ of CVD was 1.25 (95\% confidence interval (Cl): 1.06-1.48) for HDL-C concentrations $<1.04 \mathrm{mmol} / \mathrm{L}$ and 1.80 (95\% Cl: 1.56-2.07) for HDL-C concentrations $>2.07 \mathrm{mmol} / \mathrm{L}$, relative to the lowest risk group (HDL-C concentrations of 1.30$1.42 \mathrm{mmol} / \mathrm{L})$. In participants with $\mathrm{DM}$, higher HDL-C concentrations were associated with a higher risk of $C V D$, in a dose-response manner $\left(P_{\text {nonlinearity }}=0.44 ; P_{\text {trend }}<0.001\right)$. The adjusted HR of CVD was 1.63 (95\%Cl: 1.20-2.20) for HDL-C concentrations $>2.07 \mathrm{mmol} / \mathrm{L}$, relative to HDL-C concentrations of 1.30 $1.42 \mathrm{mmol} / \mathrm{L}$.

Conclusion: High HDL-C concentrations were paradoxically associated with increased risk of composite CVD outcomes in individuals with or without DM. However, low HDL-C concentrations failed to predict future CVD risk in individuals with DM.

\section{Background}

Although low density lipoprotein cholesterol (LDL-C) lowering substantially reduced the risk of cardiovascular diseases (CVD)[1], low concentrations of high density lipoprotein cholesterol (HDL-C) contributed to the residual risk of CVD even after the achievement of LDL-C concentrations at the guideline-recommended levels[1]. Experimental studies reported that HDL-C could be cardio-protective due to its effect of reverse cholesterol transport [2], inhibition of LDL oxidation[3] and reactive oxygen species[4, 5], inhibition of adhesion molecule expression[6], antiapoptotic[7], anticoagulant[8, 9], antiinfectious[10] and vasodilatory actions[11]. Consistently, previous observational studies reported an inverse or a U-shaped association between HDL-C concentrations and CVD morbidity and mortality in general populations $[12,13]$. However, clinical trials showed that significant increases in HDL-C concentrations due to the cholesteryl ester transfer protein inhibitors and other lipid modifying drugs (e.g., fenofibrate and niacin) failed to reduce future CVD risk[14-17]. This raises the question that HDL-C concentrations may not be a good biomarker for HDL function, which could be more relevant to CVD risk. 
Interestingly, experimental studies have found that the functionality of HDL may be lost in the diabetes mellitus (DM) state[18], suggesting that DM may diminish the predictive capability of HDL-C, which has not been examined in a large-scale epidemiologic studies[19]. We thus prospectively compared the association between HDL-C concentrations and CVD risk in the presence or absence of DM in a community-based cohort consisting of 91,354 Chinese adults. These participants were followed for over 10 years. Lipid parameters and covariates were repeatedly measured every 2 years, which enables us to capture the long-term lipid patterns in these individuals.

\section{Methods}

Study Populations The design and methods of the Kailuan study have been described previously[20]. This is a prospective, community-based study of 101,510 Chinese adults $(81,110$ men and 20,400 women) aged 18 years or older who were residents of the Kailuan community in Tangshan, Hebei, China in 2006. These participants were recruited from 11 hospitals affiliated with the Kailuan community and were followed biennially. In 2006 and 2007, participants completed a baseline survey including a detailed health, medical and lifestyle questionnaire and a structured physical examination, and provided a blood sample for laboratory analyses. Participants were reexamined biennially in 2008, 2010, 2012 and 2014. There were no entry criteria regarding lipid profiles concentrations. Individuals were excluded from this analysis for the presence of atherosclerotic CVD or cancer $(n=4,114)$, a lack of information on HDL-C concentrations or DM status $(n=4,144)$ at baseline, and use of lipid-lowering drugs $(n=1,898)$. The final sample comprised of 91,354 participants. Ascertainment of lipid profiles and DM status Fasting (8-12 hours) venous blood samples were collected by venipuncture into vacuum tubes containing EDTA at baseline and the subsequent biannual follow-up visits. Blood samples were stored at $-80^{\circ} \mathrm{C}$ and were analyzed in a blinded manner at the Central Laboratory of Kailuan General Hospital. Fasting serum concentrations of lipid profiles, creatinine and glucose were measured on an auto-analyzer (Hitachi 747; Hitachi, Tokyo, Japan). Fasting blood glucose concentrations were determined with hexokinase/glucose6-phosphate dehydrogenase method (BioSino Bio-technology and Science Inc., Beijing, China). The coefficient of variation using blind quality control specimens was $<2.0 \%$ for fasting blood glucose. Fasting HDL-C and LDL-C concentrations were measured with direct test method (Mind Bioengineering Co. Ltd, Shanghai, China) and with upper limits of detection of 12.90 and $3.88 \mathrm{mmol} / \mathrm{L}$, respectively[21]. Triglyceride with enzymatic colorimetric method (Mind Bioengineering Co. Ltd, Shanghai, China), and creatinine with sarcosine oxidase assay method (BioSino Bio-technology and Science Inc., Beijing, China). The intra- and inter-assay variable coefficients for each measurement were $<10 \%$. Information on physician diagnosed DM and use of glucose lowering medication was collected via a questionnaire in 2006 and was updated every two years during the follow-up. Individuals who were considered DM in the current analysis is they had a fasting blood glucose concentration of $\geq 7 \mathrm{mmol} / \mathrm{L}$, physician-diagnosed DM or self-reported use of glucose lowering medication[22]. We also conducted a secondary analysis using updated DM information during the follow-up. Ascertainment of incident death and CVD events The primary endpoint of interest was a composite of myocardial infarction, ischemic stroke or hemorrhage stroke. All death and CVD events and vital status were identified by directly contacting participants' 
family, or reviewing medical records or death certificates at the Municipal Social Insurance Institution and all the 11 Kailuan Hospitals' Discharge Register, which included all the Kailuan study participants.

Information regarding past medical history of CVD, coded as International Classification of Diseases-10th Revision was collected via biennial questionnaire since 2006[23]. Specifically, myocardial infarction was diagnosed based on cardiac symptoms, positive cardiac biomarkers or electrocardiography[24]. Ischemic stroke and hemorrhagic stroke were defined as neurological deficit of cerebrovascular cause that lasted more than 24 hours or a significant lesion detected by computed tomography or magnetic resonance imaging[25]. A blinded panel of three experienced cardiologists reviewed the medical records, discharge summaries and death certificates from local hospitals or vital statistics offices. Assessment of potential covariates Age, sex, lifestyle (e.g., cigarette smoking, alcohol consumption and habitual physical activity), family history of CVD and socio-demographic data (e.g., education level, occupation and monthly salary) were collected in 2006 and updated every 2 years using questionnaires, as detailed previously[26]. Cigarette smoking was divided into 4 categories: "never", "former", "occasional (current smoking $<1$ cigarette/d)," or "daily (current smoking $\geq 1$ cigarette/d)". Alcohol intake was assessed by a questionnaire in 2006 and participants were categorized as never, former, occasional (current drinking $<1$ time/day) or daily (current drinking $\geq 1$ time/day). Physical activity was captured by questionnaire according to activities associated with occupation and leisure time and was classified as "inactive", "moderately active" and "vigorously active"[26]. Blood pressure, waist circumference and anthropometric measurements (weight and height) were measured according to standardized procedures by trained study staff[20]. Body mass index was calculated by dividing weight in kilograms by the square of the height in meters. Blood pressure was measured with a random zero sphygmomanometer after individuals had been seated quietly for at least $5 \mathrm{~min}$. Hypertension was defined as systolic blood pressure of $\geq 140 \mathrm{mmHg}$ or diastolic blood pressure of $\geq 90 \mathrm{mmHg}$ or a history of physician- diagnosed hypertension or if on anti-hypertensive agents; pre-hypertension was defined as systolic blood pressure of 120$139 \mathrm{mmHg}$ or diastolic blood pressure of $80-90 \mathrm{mmHg}$; normotension was defined as systolic blood pressure of $<120 \mathrm{mmHg}$ and diastolic blood pressure of $<80 \mathrm{mmHg}$ [27]. High sensitivity C-reactive protein concentrations were measured using a high-sensitivity particle- enhanced immunonephelometry assay (Cias Latex CRP-H, Kanto Chemical Co. Inc., Japan)[20]. Estimated glomerular filtration rate was calculated according to the Chronic Kidney Disease Epidemiology Collaboration equation considering creatinine, sex, and age[28]. Statistical analysis The person-time for each participant was accumulated from the finishing date of the baseline survey to whichever came first: CVD events, death or termination of follow-up (December 31, 2017). The cumulative average concentrations of HDL-C and other continuous variables were used as a primary exposure because they represent long-term patterns of individuals[29]. For example, we used the $2006 \mathrm{HDL}-\mathrm{C}$ concentrations to predict outcomes occurring from 2006 to 2008, the average of the 2006 and $2008 \mathrm{HDL}-\mathrm{C}$ concentrations to predict outcomes occurring from 2008 to 2010, the average of the 2006, 2008, and $2010 \mathrm{HDL}-\mathrm{C}$ concentrations to predict outcomes occurring from 2010 to 2012, the average of the 2006, 2008, 2010 and $2012 \mathrm{HDL}-\mathrm{C}$ concentrations to predict outcomes occurring from 2012 to 2014, and the average of the 2006, 2008, 2010, 2012 and 2014 HDL-C concentrations to predict outcomes occurring after 2014. The linearity of HDL-C for mortality and CVD risk stratified by DM status was assessed using restricted cubic spline Cox model. We chose 5 knots 
based on the 5th, 27.5th, 50th, 72.5th and 95th percentiles of HDL-C concentrations that can offer adequate fit of the model. The association between HDL-C concentrations and CVD events was further examined using Cox regression analysis with corresponding 95\% confidence intervals (Cls) based on predefined groups with clinically meaningful cut-offs of HDL-C concentrations and deciles. HDL-C concentrations were categorized as $<1.04,1.04$ to $1.30,1.30$ to $1.42,1.43$ to $1.55,1.55$ to $1.81,1.81$ to 2.07 and $>2.07 \mathrm{mmol} / \mathrm{L}[30,31]$. Reference groups were selected as the ones with the lowest hazard ratios (HRs) of CVD outcomes in the spline Cox regression model. All analyses were adjusted for age, sex, body mass index, waist circumference, cigarette smoking, alcohol consumption, monthly income, education, occupation, physical activity, baseline blood pressure status, systolic blood pressure and diastolic blood pressure during follow-up, family history of myocardial infarction, stroke, hypertension or DM, estimated glomerular filtration rate, circulating concentrations of LDL-C, triglyceride, glucose and high sensitivity Creactive protein. Joint effects of HDL-C concentrations (5 groups) and DM status (yes/no) were further examined in a secondary analysis. Interactions of HDL-C/DM, with age, sex, cigarette smoking, hypertension and triglyceride, in relation to CVD were assessed by likelihood ratio testing, adjusting for aforementioned covariates. Several sensitivity analyses were conducted to test the robustness of results. Because circulating HDL-C concentrations may fluctuate due to impending CVD events and yield reverse causal association, we excluded incident CVD events occurring during the first 2 years of follow-up. Given that potential pharmacologic effect might confound the results, in a separate sub-group analysis, we excluded the participants who used glucose lowering drugs $(n=3,618)$. Statistical analyses were performed using STATA12.0 (STATA Institute) and a 2-sided $p$ value $<0.05$ was considered significant.

\section{Results}

Baseline characteristics for individuals with or without DM separately are shown in Table 1 and further divided by HDL-C concentrations cut-points in ESM Table 1 \&2. Individuals with high HDL-C concentrations were more likely to be older, women, drinker, to have hypertension, low body mass index and waist circumference, high level of systolic blood pressure and monthly salary and low concentrations of triglyceride and high sensitivity C-reactive protein. In individuals without DM, high HDL-C concentrations were associated with low concentrations of fasting blood glucose and high proportion of white collars. In contrast, high HDL-C concentrations were associated with high level of diastolic blood pressure, low level of education and physical activity and low proportion of white collars in diabetic people.

During a mean follow-up period of 10.4 years, we identified 5,076 new cases of CVD. Presence of DM significantly modified the association between HDL-C concentrations and CVD risk $\left(P_{\text {interaction }}=0.003\right)$. There was a U-shaped association between HDL-C concentrations and composite CVD outcomes in individuals without DM $\left(\mathrm{P}_{\text {nonlinearity }}<0.001\right.$; Fig. 1$)$. The HDL-C concentration of $1.33 \mathrm{mmol} / \mathrm{L}(95 \% \mathrm{Cl}$ : 1.30-1.36) was at the lowest CVD risk. Both low (<1.04 mmol/L, adjusted HR: $1.25,95 \% \mathrm{Cl}: 1.06-1.48)$ and high (>2.07 mmol/L, adjusted HR: 1.80, 95\% Cl: 1.56-2.07) concentrations of HDL-C were associated with CVD risk, relative to the HDL-C concentrations with the lowest risk of CVD (1.30-1.42 mmol/L) in 
individuals without DM (Fig. 2). In contrast, a dose-response association between high HDL-C concentrations and high CVD risk was observed in the presence of DM $\left(\mathrm{P}_{\text {nonlinearity }}=0.44 ; \mathrm{P}_{\text {trend }}<0.001\right.$; Fig. 1). High HDL-C concentrations (>2.07 mmol/L), rather than low HDL-C concentrations, were associated with increased risk of CVD in individuals with DM (adjusted HR: 1.63, 95\% Cl: 1.20-2.20) compared to the intermediate HDL-C concentrations $(1.30-1.42 \mathrm{mmHg}$ ) (Fig. 2). These results were further confirmed using a decile-based analysis.

As for CVD subtypes (e.g., myocardial infarction, ischemic and hemorrhage stroke), only the association between HDL-C concentrations and ischemic stroke followed a U-shaped curve while low HDL-C concentrations failed to predict myocardial infarction or hemorrhagic stroke occurrence in individuals without DM (ESM Fig. 1-4). The association between HDL-C concentrations and CVD risk did not materially change when we excluded participants who developed CVD events during the first 2 years of follow-up (ESM Fig. 5) or using updated DM information (ESM Fig. 6) or those who used glucoselowering drugs (only in diabetic people) (ESM Fig. 7). There were no significant interactions between HDLC/DM and age, sex, smoking and hypertension in relation to CVD risk ( $P_{\text {interaction }}>0.1$ for all).

\section{Discussion}

In our large community-based prospective study including 91,354 Chinese adults during a mean of 10.4 years of follow-up, we found that the presence and absence of DM modified the association between circulating HDL-C concentrations and future CVD risk, after adjusting for conventional confounders. A dose-response association between high HDL-C concentrations and high CVD risk was observed in participants with DM. In contrast, in participants without DM, high CVD risk was observed in low and high HDL-C concentrations groups.

HDL-C has long been regarded as the "good" cholesterol. An inverse association between HDL-C concentrations and CVD risk has been reported previously[32]. However, recent studies implied the association between HDL-C concentrations and CVD events might not be linear through the full range of HDL-C concentrations, but follows a U-shaped curve[12, 33]. In these studies, the HDL-C concentrations with lowest mortality risk ranged from 0.8 to $2.4 \mathrm{mmol} / \mathrm{L}$, which covered that in our study $(1.33 \mathrm{mmol} / \mathrm{L}$, $95 \% \mathrm{Cl}: 1.30-1.36 \mathrm{mmol} / \mathrm{L})$. The potential mechanism interpreting the U-shaped association between HDL$\mathrm{C}$ concentrations and CVD risk remains unclear. HDL-C may have a biphasic effect, as high HDL concentrations paradoxically enhanced senescence of endothelial progenitor cells and impaired tube formation[34]. HDL-C exhibited predictive capability in a certain range of concentrations, below or beyond which higher may result in adverse outcomes. The 2019 ESC/EAS Guidelines for the management of dyslipidaemias indicated that cardiovascular hazard of an extreme high HDL-C concentrations that are inappropriate for risk assessment[35].

Our findings found that DM attenuated the salutary effect of HDL-C. These results may partly interpret finding from recent clinical trials that increasing circulating HDL-C concentrations did not lower the CVD risk in high-risk population[14-16]. The relationship of HDL-C concentrations with CVD could be modified 
by hyperglycemia and insulin resistance, making it a poorer protector in individuals with DM. Of note, recent experimental studies found that hyperglycemia may modify the HDL function [36]. Increased glycation/glycoxidation of HDL impairs its reverse cholesterol transport ability and diminishes its antiatherogenic capacity[37]. Circulating HDL-C concentrations represent the sum of multiple HDL subpopulations, each with different biological capacities, particularly in special states such as DM. This suggested that the hazard of diabetic dyslipidemia for CVD risk might be better explained by number and size of circulating HDL particles rather than HDL-C concentrations [38]. DM is related to a profound redistribution of cholesterol between HDL and LDL particles, towards less of the larger, atheroprotective HDL subpopulations, and more of the smaller, lipid poor HDL particles generated, which could promote diabetic macrovascular complications [36]. Merely increasing circulating concentrations of HDL-C by pharmacological interventions or genetic conditions without any improvement of their function (e.g., cholesterol flux) and beneficial particles may not reduce CVD risk. In the Action to Control Cardiovascular Risk in Diabetes (ACCORD) trial, recruiting 5518 patients with DM, pharmaceutic raising HDL-C concentrations did not reduce the CVD risk compared to simvastatin alone[39]. The CVD benefit was observed only in the subgroup of individuals with high triglyceride and low HDL-C concentrations, which was largely ascribe to lowering hypertriglyceridemia rather than raising HDL-C concentrations.

Strengths of the present study included a large sample size and a relative long follow-up period allow us to assess the capability of the joint effect of HDL-C concentrations and DM on the risk of CVD events. Cumulative average, based on repeated measures of lipid profiles at baseline and every 2 years during the follow-up period, could reduce the within-person assay fluctuations and increase power to identify association independent of other confounders. Our study likewise has several limitations. We did not measure the HDL subclass distribution and the number and size of HDL particles, which are more closely aligned with HDL function. However, there remains a lack of cost-effective method measuring the size and composition of HDL particles in large-scale population study. Second, we did not collect information regarding DM subtype and thus cannot distinguish between type 1 and type $2 \mathrm{DM}$. However, the prevalence of type $1 \mathrm{DM}$ was low and excluding individuals with any hypoglycemic treatment did not change results materially. Third, the observational nature of our study makes it difficult to determine the causal relationship of HDL-C concentrations and CVD outcomes. A possibility of bias due to potential unmeasured confounders could not be completely removed. Randomized clinical trials, with greater follow-up may provide higher quality evidence for HDL-based approach feasible in individuals with DM. Although the pharmacological tools currently available to improve HDL function in individuals with DM remained limited, recently developed apolipoprotein mimetics and reconstituted HDL hold much promise for CVD therapeutic strategy in individuals with DM[40-42]. Fourth, women may be underrepresented $(\sim 20 \%)$ in the Kailuan study, which challenged in generalizing these results to women although we did not find a significant interaction between sex and HDL-C on mortality and CVD risk.

\section{Conclusion}

The relationship between HDL-C concentrations and CVD appeared to be modified by DM status. High HDL-C concentrations were paradoxically associated with increased risk of composite CVD outcomes in 
individuals with or without DM. However, low HDL-C concentrations failed to predict future CVD risk in individuals with DM. Further prospective studies conducted in populations with different ethnic groups are needed to confirm replicate our results.

\section{Abbreviations}

LDL-C: Low density lipoprotein cholesterol; CVD: cardiovascular diseases; HDL-C: high density lipoprotein cholesterol; DM: diabetes mellitus; Cl: confidence interval; HR: hazards ratio; ACCORD: Action to Control Cardiovascular Risk in Diabetes.

\section{Declarations}

\section{Acknowledgements}

None.

\section{Funding}

The study was supported by the Shanghai Municipal Education Commission- Gaofeng Clinical Medicine Grant Support (20172004).

\section{Consent for publication}

Not applicable.

\section{Conflict of Interest}

No authors reported any disclosures.

\section{Authors' contributions}

ZW, ZH, AL, SW and XG designed the study; CJ, SC, and FH performed the analyses; ZW, SC, SW and XG interpreted the results and wrote the manuscript; All authors read and approved the final manuscript.

\section{Availability of data and materials}

The data can be available from the corresponding authors on reasonable request.

\section{Ethics approval and consent to participate}

The study protocol was approved by the ethics committee of Kailuan Hospital and followed the guidelines of the World Medical Association Declaration of Helsinki. Written informed consent was obtained from all the participants.

\section{References}


1. Grundy SM, Stone NJ, Bailey AL, Beam C, Birtcher KK, Blumenthal RS, et al. 2018 AHA/ACC/AACVPR/AAPA/ABC/ACPM/ADA/AGS/APhA/ASPC/NLA/PCNA Guideline on the Management of Blood Cholesterol. Circulation. 2018; CIR0000000000000625.

2. von Eckardstein A, Nofer JR, Assmann G. High density lipoproteins and arteriosclerosis. Role of cholesterol efflux and reverse cholesterol transport. Arterioscler Thromb Vasc Biol. 2001; 21:13-27.

3. Robbesyn F, Garcia V, Auge N, Vieira O, Frisach MF, Salvayre R, et al. HDL counterbalance the proinflammatory effect of oxidized LDL by inhibiting intracellular reactive oxygen species rise, proteasome activation, and subsequent NF-kappaB activation in smooth muscle cells. FASEB J. 2003; 17:743-5.

4. Navab M, Hama SY, Cooke CJ, Anantharamaiah GM, Chaddha M, Jin L, et al. Normal high density lipoprotein inhibits three steps in the formation of mildly oxidized low density lipoprotein: step 1 . J Lipid Res. 2000; 41:1481-94.

5. Navab M, Hama SY, Anantharamaiah GM, Hassan K, Hough GP, Watson AD, et al. Normal high density lipoprotein inhibits three steps in the formation of mildly oxidized low density lipoprotein: steps 2 and 3. J Lipid Res. 2000; 41:1495-508.

6. Nicholls SJ, Dusting GJ, Cutri B, Bao S, Drummond GR, Rye KA, et al. Reconstituted high-density lipoproteins inhibit the acute pro-oxidant and proinflammatory vascular changes induced by a periarterial collar in normocholesterolemic rabbits. Circulation. 2005; 111:1543-50.

7. Sugano M, Tsuchida K, Makino N. High-density lipoproteins protect endothelial cells from tumor necrosis factor-alpha-induced apoptosis. Biochem Biophys Res Commun. 2000; 272:872-6.

8. Chen LY, Mehta JL. Inhibitory effect of high-density lipoprotein on platelet function is mediated by increase in nitric oxide synthase activity in platelets. Life Sci. 1994; 55:1815-21.

9. Calabresi L, Gomaraschi M, Franceschini G. Endothelial protection by high-density lipoproteins: from bench to bedside. Arterioscler Thromb Vasc Biol. 2003; 23:1724-31.

10. Nofer JR, van der Giet M, Tolle M, Wolinska I, von Wnuck Lipinski K, Baba HA, et al. HDL induces NOdependent vasorelaxation via the lysophospholipid receptor S1P3. J Clin Invest. 2004; 113:569-81.

11. Drew BG, Fidge NH, Gallon-Beaumier G, Kemp BE, Kingwell BA. High-density lipoprotein and apolipoprotein $\mathrm{Al}$ increase endothelial NO synthase activity by protein association and multisite phosphorylation. Proc Natl Acad Sci U S A. 2004; 101:6999-7004.

12. Madsen CM, Varbo A, Nordestgaard BG. Extreme high high-density lipoprotein cholesterol is paradoxically associated with high mortality in men and women: two prospective cohort studies. Eur Heart J. 2017; 38:2478-86.

13. Hirata A, Sugiyama D, Watanabe M, Tamakoshi A, Iso H, Kotani K, et al. Association of extremely high levels of high-density lipoprotein cholesterol with cardiovascular mortality in a pooled analysis of 9 cohort studies including 43,407 individuals: The EPOCH-JAPAN study. J Clin Lipidol. 2018; 12:674-84 e5.

14. Barter PJ, Caulfield M, Eriksson M, Grundy SM, Kastelein JJ, Komajda M, et al. Effects of torcetrapib in patients at high risk for coronary events. N Engl J Med. 2007; 357:2109-22. 
15. Lincoff AM, Nicholls SJ, Riesmeyer JS, Barter PJ, Brewer HB, Fox KAA, et al. Evacetrapib and Cardiovascular Outcomes in High-Risk Vascular Disease. N Engl J Med. 2017; 376:1933-42.

16. Schwartz GG, Olsson AG, Abt M, Ballantyne CM, Barter PJ, Brumm J, et al. Effects of dalcetrapib in patients with a recent acute coronary syndrome. N Engl J Med. 2012; 367:2089-99.

17. Investigators A-H, Boden WE, Probstfield JL, Anderson T, Chaitman BR, Desvignes-Nickens P, et al. Niacin in patients with low HDL cholesterol levels receiving intensive statin therapy. $\mathrm{N}$ Engl $\mathrm{J}$ Med. $2011 ; 365: 2255-67$.

18. Sorrentino SA, Besler C, Rohrer L, Meyer M, Heinrich K, Bahlmann FH, et al. Endothelial-vasoprotective effects of high-density lipoprotein are impaired in patients with type 2 diabetes mellitus but are improved after extended-release niacin therapy. Circulation. 2010; 121:110-22.

19. Navab M, Hama SY, Hough GP, Subbanagounder G, Reddy ST, Fogelman AM. A cell-free assay for detecting HDL that is dysfunctional in preventing the formation of or inactivating oxidized phospholipids. J Lipid Res. 2001; 42:1308-17.

20. Wu Z, Huang Z, Jin W, Rimm EB, Lichtenstein AH, Kris-Etherton PM, et al. Peripheral Inflammatory Biomarkers for Myocardial Infarction Risk: A Prospective Community-Based Study. Clin Chem. 2017; 63:663-72.

21. Huang S, Li J, Shearer GC, Lichtenstein AH, Zheng X, Wu Y, et al. Longitudinal study of alcohol consumption and HDL concentrations: a community-based study. Am J Clin Nutr. 2017; 105:905-12.

22. Inzucchi SE, Bergenstal RM, Buse JB, Diamant M, Ferrannini E, Nauck M, et al. Management of hyperglycemia in type 2 diabetes: a patient-centered approach: position statement of the American Diabetes Association (ADA) and the European Association for the Study of Diabetes (EASD). Diabetes Care. 2012; 35:1364-79.

23. Barta A. ICD-10-CM official coding guidelines. J AHIMA. 2009; 80:70-1.

24. Tunstall-Pedoe H, Kuulasmaa K, Amouyel P, Arveiler D, Rajakangas AM, Pajak A. Myocardial infarction and coronary deaths in the World Health Organization MONICA Project. Registration procedures, event rates, and case-fatality rates in 38 populations from 21 countries in four continents. Circulation. 1994; 90:583-612.

25. Stroke--1989. Recommendations on stroke prevention, diagnosis, and therapy. Report of the WHO Task Force on Stroke and other Cerebrovascular Disorders. Stroke. 1989; 20:1407-31.

26. Wu Z, Huang Z, Wu Y, Huang S, Wang Y, Zhao H, et al. Sedentary time, metabolic abnormalities, and all-cause mortality after myocardial infarction: A mediation analysis. Eur J Prev Cardiol. 2018; 2047487318804611.

27. Chobanian AV, Bakris GL, Black HR, Cushman WC, Green LA, Izzo JL, Jr., et al. The Seventh Report of the Joint National Committee on Prevention, Detection, Evaluation, and Treatment of High Blood Pressure: the JNC 7 report. JAMA. 2003; 289:2560-72.

28. Levey AS, Stevens LA, Schmid CH, Zhang YL, Castro AF, 3rd, Feldman HI, et al. A new equation to estimate glomerular filtration rate. Ann Intern Med. 2009; 150:604-12. 
29. Ma C, Gurol ME, Huang Z, Lichtenstein AH, Wang X, Wang Y, et al. Low-density lipoprotein cholesterol and risk of intracerebral hemorrhage: A prospective study. Neurology. 2019; 93:e445-e57.

30. Chapman MJ, Ginsberg HN, Amarenco P, Andreotti F, Boren J, Catapano AL, et al. Triglyceride-rich lipoproteins and high-density lipoprotein cholesterol in patients at high risk of cardiovascular disease: evidence and guidance for management. Eur Heart J. 2011; 32:1345-61.

31. Li X, Guan B, Wang Y, Tse G, Zou F, Khalid BW, et al. Association between high-density lipoprotein cholesterol and all-cause mortality in the general population of northern China. Sci Rep. 2019; 9:14426.

32. Gordon T, Castelli WP, Hjortland MC, Kannel WB, Dawber TR. High density lipoprotein as a protective factor against coronary heart disease. The Framingham Study. Am J Med. 1977; 62:707-14.

33. Ko DT, Alter DA, Guo H, Koh M, Lau G, Austin PC, et al. High-Density Lipoprotein Cholesterol and Cause-Specific Mortality in Individuals Without Previous Cardiovascular Conditions: The CANHEART Study. J Am Coll Cardiol. 2016; 68:2073-83.

34. Huang CY, Lin FY, Shih CM, Au HK, Chang YJ, Nakagami H, et al. Moderate to high concentrations of high-density lipoprotein from healthy subjects paradoxically impair human endothelial progenitor cells and related angiogenesis by activating Rho-associated kinase pathways. Arterioscler Thromb Vasc Biol. 2012; 32:2405-17.

35. Mach F, Baigent C, Catapano AL, Koskinas KC, Casula M, Badimon L, et al. 2019 ESC/EAS Guidelines for the management of dyslipidaemias: lipid modification to reduce cardiovascular risk. Eur Heart J. 2019;

36. Kontush A, Chapman MJ. Functionally defective high-density lipoprotein: a new therapeutic target at the crossroads of dyslipidemia, inflammation, and atherosclerosis. Pharmacol Rev. 2006; 58:342-74.

37. Hoang A, Murphy AJ, Coughlan MT, Thomas MC, Forbes JM, O'Brien R, et al. Advanced glycation of apolipoprotein A-I impairs its anti-atherogenic properties. Diabetologia. 2007; 50:1770-9.

38. Zheng C, Aikawa M. High-density lipoproteins: from function to therapy. J Am Coll Cardiol. 2012; 60:2380-3.

39. Group AS, Ginsberg HN, Elam MB, Lovato LC, Crouse JR, 3rd, Leiter LA, et al. Effects of combination lipid therapy in type 2 diabetes mellitus. N Engl J Med. 2010; 362:1563-74.

40. Nissen SE, Tsunoda T, Tuzcu EM, Schoenhagen P, Cooper CJ, Yasin M, et al. Effect of recombinant ApoA-I Milano on coronary atherosclerosis in patients with acute coronary syndromes: a randomized controlled trial. JAMA. 2003; 290:2292-300.

41. Patel S, Drew BG, Nakhla S, Duffy SJ, Murphy AJ, Barter PJ, et al. Reconstituted high-density lipoprotein increases plasma high-density lipoprotein anti-inflammatory properties and cholesterol efflux capacity in patients with type 2 diabetes. J Am Coll Cardiol. 2009; 53:962-71.

42. Morgantini C, Imaizumi S, Grijalva V, Navab M, Fogelman AM, Reddy ST. Apolipoprotein A-I mimetic peptides prevent atherosclerosis development and reduce plaque inflammation in a murine model of diabetes. Diabetes. 2010; 59:3223-8. 


\section{Tables}

Page 12/18 
Table 1: Baseline characteristics based on baseline diabetes mellitus status in the Kailuan Study

\begin{tabular}{|c|c|c|}
\hline Characteristics & Without DM & With DM \\
\hline Number & 83,110 & 8,244 \\
\hline Age, year * & $50.0(12.4)$ & $55.8(10.6)$ \\
\hline Men, \% & 79.2 & 82.7 \\
\hline $\mathrm{BMI}, \mathrm{kg} / \mathrm{m}^{2}$ & $24.9(3.12)$ & $25.8(3.09)$ \\
\hline $\mathrm{WC}, \mathrm{mm}$ & $86.9(8.31)$ & $90.2(8.12)$ \\
\hline $\mathrm{TG}, \mathrm{mmol} / \mathrm{L}$ & $1.63(1.23)$ & $2.07(1.70)$ \\
\hline Glucose, $\mathrm{mmol} / \mathrm{L}$ & $5.34(0.85)$ & $9.07(2.68)$ \\
\hline $\mathrm{hs}-\mathrm{CRP}, \mathrm{mg} / \mathrm{L}^{\dagger}$ & $0.30(1.05)$ & $0.53(1.09)$ \\
\hline $\mathrm{HDL}-\mathrm{C}, \mathrm{mmol} / \mathrm{L}$ & $1.50(0.35)$ & $1.49(0.37)$ \\
\hline LDL-C, mmol/L & $2.55(0.73)$ & $2.63(0.78)$ \\
\hline SBP, mmHg & $132.1(17.7)$ & $140.7(18.3)$ \\
\hline DBP, mmHg & $83.9(9.44)$ & $86.0(9.68)$ \\
\hline eGFR, $\mathrm{ml} / \mathrm{min} / 1.73 \mathrm{~m}^{2}$ & $88.9(19.2)$ & $84.2(20.8)$ \\
\hline \multicolumn{3}{|l|}{ Occupation, \% } \\
\hline Coalminer & 31.6 & 28.1 \\
\hline Other blue collars & 62.1 & 64.8 \\
\hline White collar & 6.36 & 7.04 \\
\hline \multicolumn{3}{|l|}{ Average income, \% } \\
\hline$<600 ¥$ per mo & 28.5 & 28.1 \\
\hline $600-800 ¥$ per mo & 57.4 & 58.3 \\
\hline $800-1000 ¥$ per mo & 7.52 & 7.03 \\
\hline$\geq 1000 ¥$ per mo & 6.58 & 6.58 \\
\hline Family history of MI, \% & 3.11 & 2.78 \\
\hline Family history of stroke, \% & 8.93 & 7.93 \\
\hline Family history of hypertension, \% & 24.6 & 21.8 \\
\hline Family history of DM, \% & 7.88 & 13.3 \\
\hline
\end{tabular}




\begin{tabular}{|c|c|c|}
\hline \multicolumn{3}{|l|}{ Smoking status, \% } \\
\hline Never & 60.5 & 61.9 \\
\hline Former & 4.92 & 7.14 \\
\hline Occasionally & 3.61 & 3.10 \\
\hline Daily & 31.0 & 27.9 \\
\hline \multicolumn{3}{|c|}{ Alcohol consumption, \% } \\
\hline Never & 59.1 & 62.3 \\
\hline Former & 3.15 & 5.47 \\
\hline Occasionally & 19.7 & 15.5 \\
\hline Daily & 18.0 & 16.8 \\
\hline \multicolumn{3}{|l|}{ Education level, \% } \\
\hline Primary & 9.78 & 13.9 \\
\hline middle/high school & 83.0 & 81.9 \\
\hline college/university & 7.24 & 4.25 \\
\hline \multicolumn{3}{|l|}{ Physical activity, \% } \\
\hline Inactive & 8.83 & 7.66 \\
\hline Moderately active & 76.6 & 73.4 \\
\hline Vigorously active & 14.5 & 19.0 \\
\hline \multicolumn{3}{|c|}{ Blood pressure status, $\%$} \\
\hline Normal & 21.5 & 9.48 \\
\hline Pre-hypertension & 37.5 & 27.9 \\
\hline Hypertension & 41.0 & 62.7 \\
\hline
\end{tabular}

Abbreviations: DM, diabetes mellitus; $\mathrm{BMI}$, body mass index; TG, triglyceride; WC, waist circumference; hs$\mathrm{CRP}$, high sensitivity C-reactive protein; HDL-C, high density lipoprotein cholesterol; LDL-C, low density lipoprotein cholesterol; SBP, systolic blood pressure; DBP, diastolic blood pressure; eGFR, estimated glomerular filtration rate; Ml: myocardial infarction. To convert from $\mathrm{mmol} / \mathrm{L}$ to $\mathrm{mg} / \mathrm{dL}$, divide by 0.0259 for HDL-C and LDL-C and 0.0113 for triglycerides.

* Continuous variables were reported as mean (standard deviation).

${ }^{\dagger}$ hs-CRP was log-transformed. 


\section{Figures}
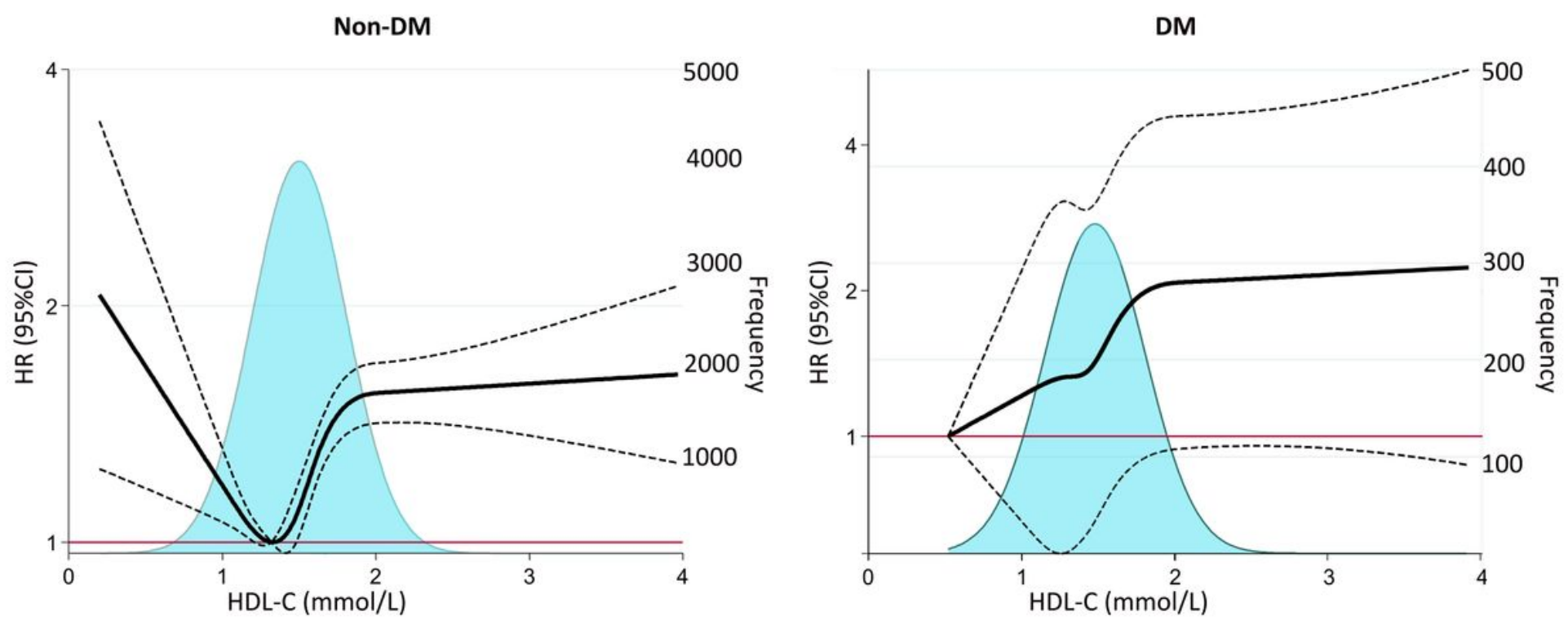

\section{Figure 1}

Adjusted hazard ratios (HRs, solid line) and 95\% confidence intervals (Cls, dashed lines) for cardiovascular diseases risk with high density lipoprotein cholesterol (HDL-C) concentrations using restricted cubic spline Cox model in individuals with or without diabetes mellitus (DM). The blue area indicates the distribution of HDL-C concentrations. All models were adjusted for age (years), sex (men or women), family history of myocardial infarction, stroke, diabetes mellitus or hypertension (yes or no), smoking status (never, former, occasionally or daily), body mass index (quartiles), waist circumference (quartiles), low density lipoprotein cholesterol (quartiles), triglyceride (quartiles), high sensitivity C-reactive protein (quartiles), systolic blood pressure (quartiles), diastolic blood pressure (quartiles), glucose (quartiles), estimated glomerular filtration rate (quartiles), alcohol consumption (never, former, occasionally or daily), educational level (primary, middle/high school or college/university), occupation (white collar, coal miner or blue collar), physical activity (inactive, moderately active, or vigorously active), monthly salary ( $<600,600-800,800-1000$ or $\geq 1000 ¥)$, baseline blood pressure status (normal, prehypertension, or hypertension). 


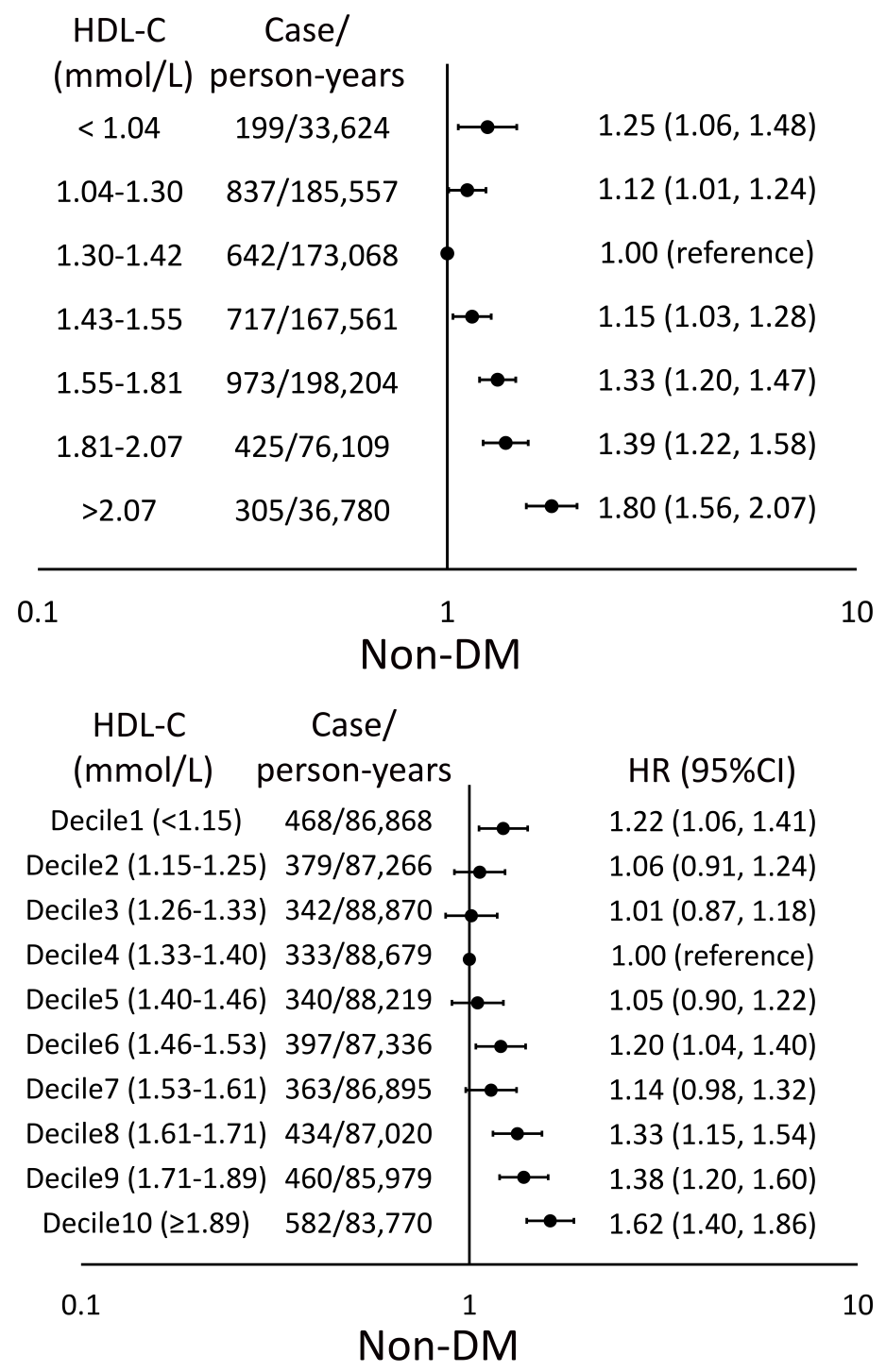

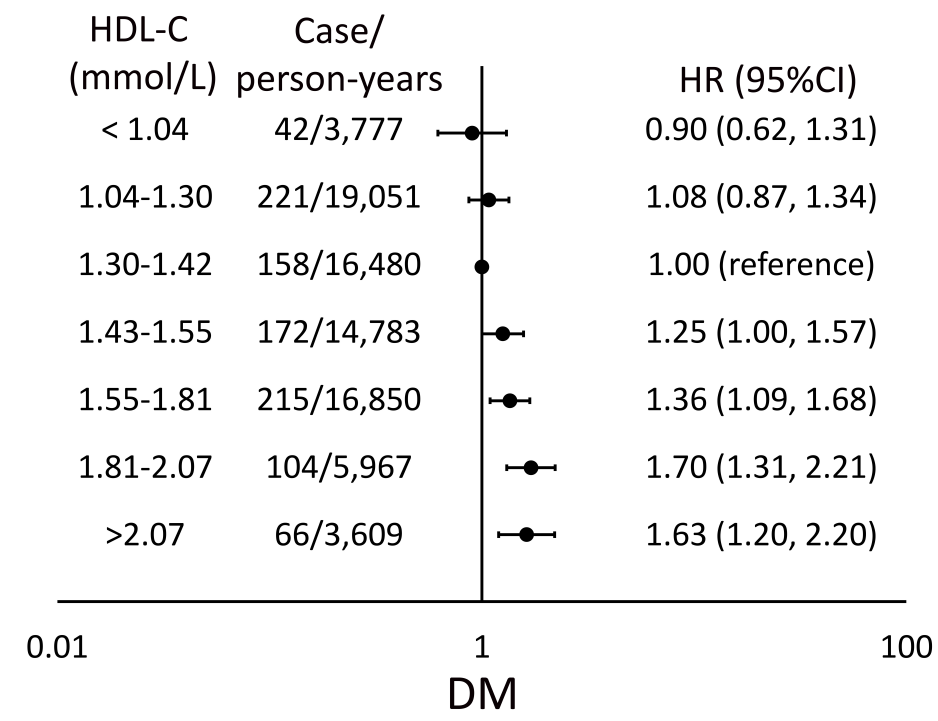

HDL-C Case/

(mmol/L) person-years

\begin{tabular}{|c|c|c|c|}
\hline \multicolumn{3}{|c|}{ (mmol/L) person-years } & HR (95\%(I) \\
\hline Decile1 (<1.13) & 80/8,091 & & $0.85(0.62,1.18)$ \\
\hline Decile2 (1.13-1.23) & $99 / 8,148$ & & $1.11(0.81,1.50)$ \\
\hline Decile3 (1.23-1.31) & $96 / 8,104$ & & $1.13(0.83,1.55)$ \\
\hline Decile4 (1.31-1.38) & $84 / 8,284$ & & 1.00 (reference) \\
\hline Decile5 (1.38-1.44) & $84 / 8,342$ & & $1.05(0.76,1.44)$ \\
\hline Decile6 (1.44-1.51) & $88 / 8,136$ & & $1.14(0.83,1.55)$ \\
\hline Decile7 (1.51-1.59) & $101 / 8,143$ & $\rightarrow$ & $1.30(0.97,1.76)$ \\
\hline Decile8 (1.59-1.70) & $93 / 8,001$ & $\leftrightarrow$ & $1.25(0.92,1.70)$ \\
\hline Decile9 (1.70-1.88) & $121 / 7,881$ & $\mapsto$ & $1.54(1.15,2.06)$ \\
\hline Decile10 ( $\geq 1.89)$ & $132 / 7,385$ & $\rightarrow$ & $1.62(1.21,2.17)$ \\
\hline
\end{tabular}

\section{Figure 2}

Adjusted hazard ratios (HRs) and 95\% confidence intervals (Cls) for cardiovascular diseases (CVD) risk based on concentration cut-points ((upper panel)) and deciles (bottom panel) of high density lipoprotein cholesterol (HDL-C) in individuals with or without diabetes mellitus (DM). Reference groups were the ones containing HDL-C concentrations with the lowest HRs of CVD in the spline Cox regression model. All models were adjusted for age (years), sex (men or women), family history of myocardial infarction, stroke, diabetes mellitus or hypertension (yes or no), smoking status (never, former, occasionally or daily), body mass index (quartiles), waist circumference (quartiles), low density lipoprotein cholesterol (quartiles), triglyceride (quartiles), high sensitivity C-reactive protein (quartiles), systolic blood pressure (quartiles), diastolic blood pressure (quartiles), glucose (quartiles), estimated glomerular filtration rate (quartiles), alcohol consumption (never, former, occasionally or daily), educational level (primary, middle/high school or college/university), occupation (white collar, coal miner or blue collar), physical activity (inactive, moderately active, or vigorously active), monthly salary ( $<600,600-800,800-1000$ or $\geq 1000 ¥)$, baseline blood pressure status (normal, pre-hypertension, or hypertension). 

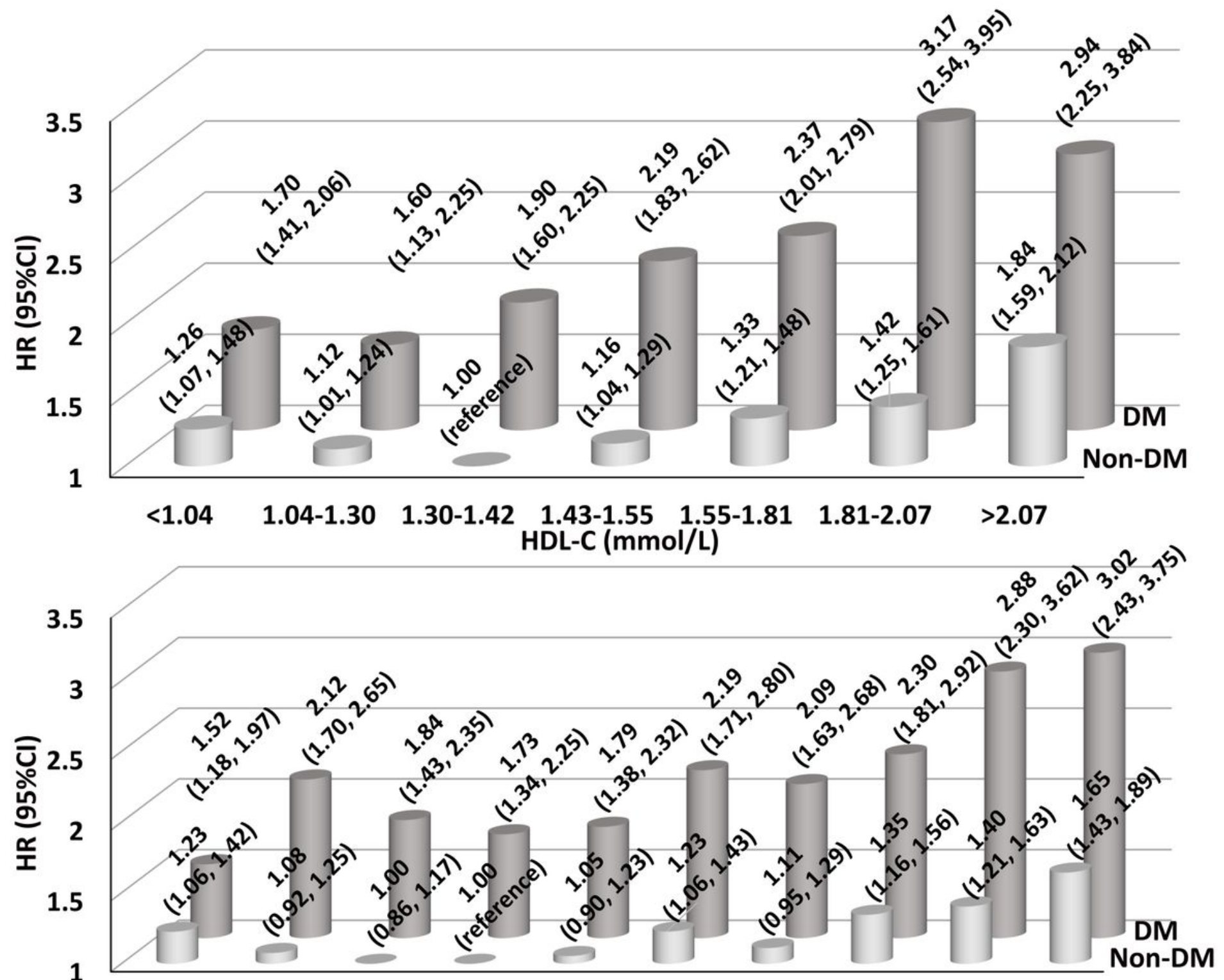

1

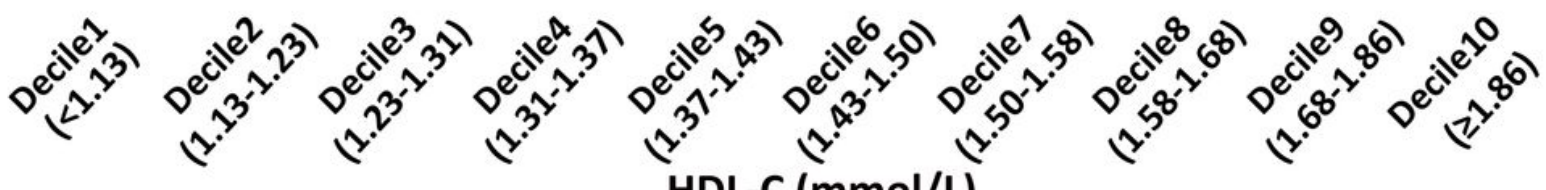

Figure 3

Adjusted hazard ratios (HRs) and 95\% confidence intervals (Cls) for cardiovascular diseases (CVD) risk among combined subgroups of high density lipoprotein cholesterol (HDL-C) clinical cut-offs and diabetes mellitus (DM) among entire cohort. The combined value of HDL-C concentrations with the lowest HRs of CVD and non-DM was used as a reference. All models were adjusted for age (years), sex (men or women), family history of myocardial infarction, stroke, diabetes mellitus or hypertension (yes or no), smoking status (never, former, occasionally or daily), body mass index (quartiles), waist circumference (quartiles), low density lipoprotein cholesterol (quartiles), triglyceride (quartiles), high sensitivity C-reactive protein (quartiles), systolic blood pressure (quartiles), diastolic blood pressure (quartiles), glucose (quartiles), estimated glomerular filtration rate (quartiles), alcohol consumption (never, former, occasionally or daily), 
educational level (primary, middle/high school or college/university), occupation (white collar, coal miner or blue collar), physical activity (inactive, moderately active, or vigorously active), monthly salary ( $<600$, $600-800,800-1000$ or $\geq 1000 ¥$ ), baseline blood pressure status (normal, pre-hypertension, or hypertension).

\section{Supplementary Files}

This is a list of supplementary files associated with this preprint. Click to download.

- supplementaltablesandfiguretoCD.docx

- supplementaltablesandfiguretoCD.docx

- supplementaltablesandfiguretoCD.docx 